\title{
Predicting a Failure of Public Speaking Performance
}

\section{Using Multidimensional Assessment}

Jiewen Hua ${ }^{1}$, Edith Filaire ${ }^{2}$, Tom Giraud ${ }^{3}$, David Antonio Gómez Jáuregui ${ }^{3}$, Mariette Soury ${ }^{3}$, Jean-Claude Martin ${ }^{3}$, Laurence Devillers ${ }^{4}$ and Christine Le Scanff ${ }^{1}$

1. Department of CIAMS, University of Paris-Sud, Orsay 91405, France

2. Department of CIAMS, University of Orléans, Orléans 45067, France

3. Department of LIMSI-CNRS, University of Paris-Sud, Orsay 91405, France

4. Department of LIMSI-CNRS, University of Paris-Sorbonne, Paris 75000, France

\begin{abstract}
The present study aimed to examine multidimensional factors that contribute to a poor performance in a public speaking task. An adapted version of the Trier Social Stress Test (TSST) as used to elicit psychosocial stress among 43 university students and multidimensional assessments were involved to investigate acute stress responses by psychological measures (i.e. personality, affect, appraisal, coping), physiological measures (i.e. cortisol; Dehydroepiandrosterone: DHEA; ratio of cortisol/DHEA) and behavioural measures (voice, postural control). Our results showed that psychological factors seemed to be the most sensitive to stress performance. A mediation effect was detected between psychological factors and objective performance. Cortisol to DHEA ratio also showed to be associated with speaking performance. This study added evidence to the literature with regards to a multidimensional way to study human stress response and may help individuals use functional coping to improve their performance and better adapt to stressful situations.
\end{abstract}

Key words: Public speaking performance, trait anxiety, coping, appraisal, the ratio of cortisol/DHEA.

\section{Introduction}

Understanding the cause of failure in public speaking performance would help to understand how to get prepared to cope with stressful situations for people who are looking for a job or for students who are having an exam. Researchers have paid attention to different stress performances such as sport performance [1], academic performance [2], memory performance [3] and decision making performance [4]. Though the public speaking task has been used widely in assessing human stress response, few studies have evaluated stress performance using this paradigm.

Stress reactivity is a complex phenomenon involving several response systems, namely cognitive, emotional, physiological and behavioural responses [5]. Empirical evidence indicated that motivated

Corresponding author: Christine Le Scanff, Ph.D., professor, research field: sport psychology. performance tasks containing social-evaluative threat and uncontrollable elements elicit robust and reliable psychological and biological stress responses [6]. Indeed, research suggested that psychological and physiological response have been examined wildly during the past decades in stress research [7]. However, stress performance has rarely been evaluated by a multidimensional approach which takes into account the different dimensions of a stress reaction and which one is the best predictor.

\subsection{Public Speaking Task}

The Trier Social Stress Test (TSST) [8], a standardized public speaking task developed to induce acute psychosocial stress in a laboratory setting, is the most frequently used protocol in stress research. It was considered to trigger social-evaluative threat reliably [7], leading to psychobiological stress reactions, such as the psychological response, 
cardiovascular and neuroendocrine stress reactivates $[1,6,8]$. The TSST protocol consists of a waiting period, speech preparation, speech performance and mental arithmetic performance, followed by recovery periods [9].

In the traditional TSST, performance is evaluated by a mental arithmetic task. Studies examined task performance which was achieved by reporting performance satisfaction [10]. However, it was suggested that this cognitive task induced less stress than the public speech [11]. Thus, it does not as thoroughly represent the stress performance as the public speech. For this reason, in the current study the arithmetic task in the traditional TSST was replaced by a feedback \& question session for an ecological reason: to approximate a job interview in real life situation. This adapted protocol elicited psychosocial stress with significant stimulation of the hypothalamohypophyso- adrenal and sypatho- adreno medullar axis [12].

\subsection{Psychological Factors and Performance}

Several studies suggest that personality and individual differences may influence the impact of stress [13]. Some traits such as neuroticism have been observed to intensify stress reactivity and lead to greater stress vulnerability [14, 15]. Characterized by a tendency towards negative emotional states, neuroticism was observed to be associated with psychological stress response and poor performance [1, 16-18]. Individuals who rate high on neuroticism or anxiety have a chronic tendency to worry and therefore are considered to be vulnerable in stress adaptation.

Evidence also indicates that a high level of anxiety is associated with poor academic performance [19], musical performance [20], and sport performance [21, 22]. Empirical studies suggest that physiological arousal and cognitive anxiety are significant factors leading to a low performance [23]. During public speaking tasks conducted in laboratory, anxiety was observed to be associated with greater cardiovascular responses during the performance [24, 25].

Individuals also differ in terms of stress susceptibility, which reflects on the cognitive appraisals towards stressful situation [26]. Cognitive appraisals are considered as having an effect on coping choice and thereby influence performance. Threat appraisal relates positively to task stress and cardiac reactivity [27]. Moreover, according to Baggett et al. [28], people experiencing threat appraisals report having more negative emotions and worse performances than people who do not experience threat appraisal. Thus, threat appraisal is considered to play an important role in stress performance.

Dysfunctional coping contributes to maladaptive psychological and physiological responses to acute stressors and performance. Compared to the functional coping, which helps people to deal with problems, dysfunctional coping, such as denial or behavioural disengagement, tends to allow people to avoid reality. In clinical settings, the use of passive coping strategies or focusing on emotions results in greater depression, pain, and lower general self-efficacy [29]. Although Gallagher [30] suggests a more complex personality-coping -outcome trend, the link between different types of copings and academic performance under stressor was observed.

\subsection{Physiological Markers and Performance}

Additionally, studies have considered the associations between physiological and psychological responses to the TSST, emphasizing that most stress experiences are accompanied by physiological and psychological responses [6]. Cortisol, a hormone released from the hypothalamic-pituitary-adrenal axis (HPA), has been proposed to be a reliable biomarker in examining acute psychological stressor [7]. Salivary cortisol has been widely studied in response to laboratory induced stressful tasks and it was observed to be associated with elevated stress level and psychological stress feelings [6]. Moreover, 
evidence suggested that acute stress and elevated cortisol might influence task performance [3, 5]. Dehydroepiandrosterone (DHEA), a major steroid release by the adrenal cortex, is also an important marker in examining responses to stressful situations [31]. DHEA has a regenerative role [32] and serves as a protective role against the negative effects induced by stress exposure [33, 34]. Van Niekerk et al. [35] also suggested that DHEA is associated with psychological well-being.

Other studies suggested that it is meaningful to consider the cortisol to DHEA ratio as being a reliable index when examining stress responses to situations causing social anxiety [31]. DHEA could antagonize cortisol activity [36] and plays a protective role in stress reactivity $[32,37]$. Thus, the cortisol to DHEA ratio is considered to be a marker of endocrine imbalance of the HPA axis function. Literature suggests that a low cortisol/DHEA ratio indicates a higher protection against stress and a better military performance [37]. The cortisol to DHEA ratio was also found to be linked to mental disorders such as anxiety and depression.

\subsection{Behavioural Outcomes and Performance}

There is growing evidence suggesting that human emotions are detectable from performance [38]. From an affective-computing point of view, researchers are trying to make use of video and audio measures to detect emotions in human voices [39], body movements and postural control [40]. In response to a challenging situation such as a public speaking task, behavioural changes can be expressed through voice, body gestures, and body movements etc. Voice index is an important parameter in evaluating speech performance. Evidence showed that acoustic characteristics of speech can be useful for assessing anxiety level [41, 42].

Beside the characteristics of voice, body movements also provide cues that can be useful for understanding stress response. Extracted from video recordings of the participant, Quantity of Motion (QoM) and Contraction Index (CI) are considered to recognise bodily expressions of emotion [38, 40, 43]. These indexes were observed to be associated with emotions in dance performance. According to Camurri et al. [43], CI was higher in performing negative emotions such as fear and grief, and such negative emotions related to an increasing trend of QoM. Although no direct links have been found between these indexes and performance, it seems that task performances can be influenced by negative emotions under stress, which might be detected from these behavioural indexes. In turn, we can expect a profile with such links between a bad performance and increased $\mathrm{CI}$ and QoM.

In this study, our aim was to use integrated methods to examine psychological, physiological and behavioural responses to a laboratory-induced psychosocial stressor. Such a systemic approach enables a better understanding of human emotions in a situation that elicits psychosocial stress. The general assumption is that public speaking performance is influenced by individual differences in terms of personality, appraisals and coping, and that some consequences might be detected in emotional experience, physiological reactivity and behavioural outcomes.

In the present study, we not only examined the direct links, but also explored the indirect links between the study variables and public speaking performance to have a more accurate "picture" of the stress reaction. Coping, such as avoidance, has been suggested to mediate the link between stress-sensitive trait and psychological distress [44]. Performance, as the outcome of stress, was therefore expected to be associated with both stress-sensitive trait (e.g. trait anxiety, neuroticism) and dysfunctional coping, and moreover, the link between trait and performance can be mediated by coping.

Therefore, based on the current knowledge from the literature, we put forward the hypothesis that 
participant's profile should enable predicting a failure in public speaking performance. Based on the general aims, we hypothesized that:

(1) Performance was negatively associated with psychological factors of neuroticism $(\mathrm{N})$, trait anxiety, negative affect (NA), threat appraisals and dysfunctional coping;

(2) Performance was negatively associated with physiological parameters of cortisol and the cortisol to DHEA ratio;

(3) Performance was negatively associated with behavioural markers of Contract Index (CI), Quantity of Motion (QoM), the percentage of silence, but positively associated with voice intensity;

(4) The link between trait anxiety and performance was mediated by dysfunctional coping.

\section{Method}

\subsection{Participants}

Participants were forty-three students recruited from a University in France based on their voluntary. They were 19 males and 24 females. Mean age of male students was $23.9 \pm 4.4$ years, body weight: 76.3 $\pm 10.1 \mathrm{~kg}$; height: $179.6 \pm 10.1 \mathrm{~cm}$; body mass index: $23.6 \pm 3.0 \mathrm{~kg} / \mathrm{m}^{2}$. For the female participants, the mean age was $28.2 \pm 9.9$ years, body weight: $63.0 \pm$ $10.4 \mathrm{~kg}$; height: $166.9 \pm 8.6 \mathrm{~cm}$; body mass index: $22.5 \pm 2.9 \mathrm{~kg} / \mathrm{m}^{2}$.

\subsection{Measures}

\subsubsection{Psychological Measures}

Big Five was measured using The French version of Big Five Inventory (BFI [45]; the French version: BFI-fr [46]). It is a 45-item self report questionnaire scored on a 5-point Likert scale which assesses five broad dimensions of personality, namely Neuroticism, Extraversion, Conscientiousness, Agreeableness, and Openness to Experience. Each item was assessed by a sentence starting with "I see myself someone as..." The BFI-Fr showed good psychometric properties with the Cronbach's alpha 0.82 for Neuroticism and
Extraversion, 0.75 for Agreeableness, 0.80 for Conscientiousness and 0.74 for Openness to Experience [46].

State and Trait anxiety were evaluated by Spielberger's State Trait Anxiety Inventory Form Y that consists of two 20-item scales measuring on a 4-point Likert scale: STAI-State and STAI-Trait (STAI-Y [47], the French version [48]). The "State" scale assesses how individual feel "right now, at this moment" (e.g., "I feel at upset"), whereas the "Trait" scale measures "the general feel" (e.g., "I lack self confidence"). This scale has good internal consistency. The Cronbach's alpha varies from 0.80 to 0.92 [48].

Emotional states were evaluated by Positive and Negative affect Schedule which consists of 20 items on a 5-point Likert scale (PANAS [49], French version [50]). It assess either positive (e.g., interested) or negative (e.g., guilty) emotional states each with 10 items. The Cronbach's alpha was 0.91 for PA and 0.86 for NA in the previous study [51].

Coping strategies were measured using the Brief COPE (an abbreviated version of the COPE [52], French version [53]). The Brief-COPE consists of 14 subscales each of which contains two items on a 4-point Likert scale. Considering that some items were not compatible with the context of the study (e.g. I use alcohol or other drugs to make myself feel better), three subscales were excluded: Emotional support, Self-distraction and Substance use. However, the examination on scale reliability revealed that the Cronbach's alphas were not all satisfactory for the subscales, we therefore regrouped the scales into two large dimensions: functional coping and dysfunctional coping. The functional coping consists of 12 items from active coping, planning, use of instrumental help, positive reframing, and acceptance and humour, showing an alpha coefficient of 0.76. The dysfunctional coping consists of 4 items from behaviour disengagement and self-blame, generating a Cronbach's alpha of 0.67.

Threat and challenge appraisals were measured by 
four questions [54]. The former two questions (1) and (2) assess how important and stressful participants perceive the event (i.e. how threatening) and the latter two (3) and (4) showed their efficacy in coping with the stressor. The current study used a conceptual split in line with [15]. Threatened participants perceived more threat than ability so the ratio of threat to challenge appraisal was greater than one, while challenged participants perceived more ability than threat, then the ratio of threat to challenge appraisal was less than one [15]. These four questions were translated from English to French by a French professor in psychology who has adequate English level, then revised by a bilingual (in English and French) colleague in psychology, and finally translated back to English for comparison until a satisfactory version is reached.

Subjective task evaluation was assessed by five questions [28] assessing five single dimensions on motivation, perceived frustration, stress, difficulty of the task and evaluation of own performance, e.g. to what extent did you feel frustration in this public speaking task?

Subjective and objective performances were measured for each participant. Subjective performance was assessed by a single question as described above. Objective performance was evaluated by three qualified assessors following criteria adapted from Fydrich et al. [55] and Harb et al. [56]. The performance was evaluated with respect to three aspects: the content of the speech itself (i.e. proper introduction, organization of contents, information and details, final summary of main ideas etc.), the delivery of the speech (i.e. voice quality, eye contact, appearance etc.) and the quality of the answers to the questions. Scores ranged between 1 and 5 . We observed a moderate correlation between subjective and objective performances $\left(r=0.482^{* *}\right)$ showing a good reliability of these measures.

\subsubsection{Behavioural Measures}

Participants' behavioural responses were recorded to enable future multimodal analyses. Voices of participants were recorded with a wireless microphone. Whole body movements were recorded by a video camera and a Kinect. Postural control was recorded by a force plate hidden under participants' feet. Behaviours of the assessors were also recorded by a camera. Several parameters were automatically extracted from the video file: quantity of motion (QoM) and contraction index (CI). The contraction index is the ratio of the silhouette's surface over the surface of the convex hull [38]. The quantity of motion $(\mathrm{QoM})$ is a measure of the amount of detected motion, from images carrying information about variations in the silhouette shape and position [57].

\subsubsection{Physiological Parameters and Measurements}

Saliva samples were collected using Salivette collection tubes (Sarstedt Co., Nümbrecht, Germany). The cotton roll was omitted and the salivette container was used for saliva sampling. Participants donated whole unstimulated saliva using the passive drool technique for 3 minutes. They were instructed to swallow to empty the mouth before the unstimulated whole saliva sample was collected. The collection of whole saliva by passive drool is the most reliable option, as cotton or polyester-based materials tend to increase acidity and provide false concentrations of saliva components [58]. Time of day was controlled because cortisol levels are known for small spontaneous fluctuations in the late afternoon; all tests were done between 04.00 and 07.30 p.m. Eating, and drinking beverages containing alcohol, caffeine, or fruit juices were restrained for 60 minutes before sampling. The subjects were told not to undergo intensive physical activity for the 48 hours prior to the experiment and to refrain from any sporting activities at all 24 hours before the study. Besides these restrictions, participants were free to follow their normal daily routines on the sampling days. Finally, subjects were instructed not to brush their teeth or eat at least 60 minutes before the beginning of the test.

Saliva samples were scheduled at 60 minutes after 
arrival, 3 minutes pre-stress and at 3,15, 30, and 45 minutes post-stress. Saliva samples were stored at $-45^{\circ} \mathrm{C}$ until biochemical analysis. Samples were centrifuged at $3000 \mathrm{rpm}$ for 15 minutes. The concentration of cortisol and DHEA in saliva was determined by an enzyme immunoassay using the EIA kit (Salimetrics, USA). Intra-assay maximal coefficients of variation were $3.6 \%$ and $6.8 \%$ for cortisol and DHEA, respectively. Inter-assay maximal coefficients of variation were $6.4 \%$ and $8.4 \%$ for cortisol and DHEA, respectively. Cortisol and DHEA concentration was expressed as nmol.l-1. All samples were processed in duplicate during the same assay section.

\subsection{Procedures}

Psychological, physiological and behavioural measures during the experimentation included the personality tests, self-report measures on emotional experiences, appraisal of the situation and coping, collection of saliva samples, voice and body movement detections and the evaluation of public speaking performance.

A consent form was sent by email to participants providing information about the study, confidentiality and contact information. Participants read and signed the consent form and brought it back to the researchers on the day of the experiment. Participants were also required to fill in the questionnaires of personality tests sent by email.

Each participant arriving at the laboratory was required to sit in a quiet room. After 60 minutes, the first saliva sample measuring the baseline was collected. Fig. 1 presents the schedule of the experimentation.

The instruction was given to each participant several minutes before entering the experimentation room, informing that they were going to provide a five-minute speech in front of two judges as if they were applying for a job. The instructions were as follow: "You are applying for a job that is particularly important to you. You will have five minutes to introduce yourself and describe your personal characteristics (e.g. personality, skills, and experiences) that you view as strengths and that would allow you to be considered for this job. You must also point out those characteristics that might be perceived as weaknesses and explain how you have or are willing to improve them. Be as sincere as possible. This is an important prerequisite for this position." Participants were also informed that their performance would be videotaped. After reading the instructions, participants were required to report their current emotional states and their appraisals for the coming task either as a challenge or as a threat. At the same time, they were asked to provide the second salivary sample.

Next, they entered the experimental room where light was kept constant throughout the experimental session. They were asked to stand on an "X" marked on the floor to deliver their speech in front of a video camera. Participants faced two qualified assessors who took notes while the speech was delivered by the participant. As instructed, the participant's speech was

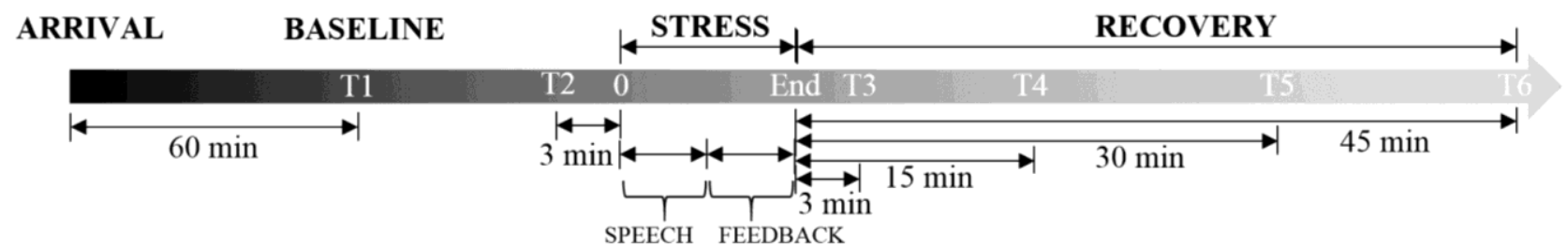

Fig. 1 Experimental timeline (Figure adapted from [12]).

T1: Saliva Sample 60 minutes after arrival at the laboratory; T2: Saliva Sample 3 minutes pre-test; pre-task subjective emotional states; T3: Saliva Sample 3 minutes post-test; post-test subjective emotional states; T4: Saliva Sample 15 minutes post-test; T5: Saliva Sample 30 minutes post-test; T6: Saliva Sample 45 minutes post-test. 
stopped after five minutes. Then the assessors provided feedback and asked questions to the participant. The two assessors showed either a negative or a positive attitude towards the participants by giving negative or positive feedback. Performance was evaluated later from the videos against criteria regarding the speech content, the way the speech was delivered and the quality of the answers to the assessors' questions. The assessors were blind to the psychological characteristics of the participants. The assessors' questions were scripted and presented in the same order for each subject, but they were also adapted to the content of the delivered speech in order to gain more credibility and to be closer to a real job interview situation.

Immediately after the task, each participant was asked to report her current mood again and her perception of the stress feeling, her motivation, the task difficulty, and her performance through self-report scales. Before leaving the experimental room, participants provided the third saliva sample and were then thanked for their participation. After leaving the experiment room, the participants remained in the laboratory for another 45 minutes and provided three other saliva samples, after which they were debriefed and allowed to leave. The whole interview session lasted for a total of 20 minutes per participant. During the experiment, a researcher supervised the scales distribution at the right timing (immediately before and after the task), making sure that the subjective emotional states of each participant were collected at given time points.

\subsection{Data Analyses}

SPSS for Windows Version 20.0 was used to analyse the data. Normality of data distribution was established for physiological variables using Kolmogorov-Smirnov test. Psychological data are presented as mean and standard deviations (SD) and all the other results are presented as mean and standard errors (SEM). Statistical significance level was set at $P<0.05$. Pearson correlation analysis was conducted to determine the relationship between public speaking performance and psychological, physiological and behavioural factors. To test the mediating effect, bootstrapping mediation analyses involving simultaneous entry regression analyses were used [59]. Bootstrapping is a computationally driven re-sampling method in which statistics (e.g. mediated effects) are calculated in multiple samples generated from the original sample. The mediation analyses were conducted using a macro for SPSS designed and developed to assess the indirect effects of multiple mediators [60].

\section{Results}

Descriptive statistics (Mean and Standard Deviation) are presented in Table 1.

Correlations were examined between objective task performance and multiple responses (Table 2). Based on the negative correlations between performance and associated factors, unfavourable profiles for the objective performance could include trait anxiety, negative affective states before and after the task, anxiety states before and after the task, ratio of threat to challenge appraisals, dysfunctional coping, and the ratio of cortisol/DHEA. The global behavioural responses detected on body movement, gesture and voice did not contribute to the correlations.

Results revealed an indirect effect between trait anxiety and performance through appraisals and coping. Within bootstrap analysis, the predictor variable of trait anxiety and the mediating variables of threat/challenge appraisals and dysfunctional coping were regressed on the dependent variable of objective performance score, producing a dependent model summary of $F(3,39)=6.42, P<0.01, R^{2}=0.33$, $R^{2}$ adjusted $=0.28$. As shown in Fig. 2, the significant, negative direct effect between trait anxiety and performance score was totally mediated by appraisals and dysfunctional coping. In particular, trait anxiety significantly predicted these two variables. In turn, 
Table 1 Mean and standard deviation (SD) of main study variables $(N=43)$.

\begin{tabular}{llll}
\hline & Mean & SD & Cronbach's alpha \\
\hline $\mathrm{N}$ & 22.07 & 6.38 & 0.91 \\
STAIt & 42.47 & 10.62 & 0.94 \\
STAIs & 42.58 & 13.80 & 0.96 \\
PA & 28.63 & 8.53 & 0.86 \\
NA & 17.23 & 9.49 & 0.96 \\
COPEdys & 7.30 & 2.60 & 0.67 \\
Threat & 7.16 & 2.41 & 0.69 \\
Challenge & 9.58 & 2.25 & 0.86 \\
Appraisal & .82 & 0.43 & - \\
SP & 2.35 & 1.11 & - \\
OP & 2.62 & 0.98 & - \\
rCDHEA3 & 15.65 & 4.2 & - \\
\hline
\end{tabular}

N: Neuroticism; STAIt: trait anxiety; STAIs: post-task states anxiety; PA: post-task positive affect; NA: post-task negative affect; COPEdys: dysfunctional coping; Threat: threat appraisals; Challenge: challenge appraisals; Appraisal: the ratio of threat appraisal to challenge the appraisal; SP: subjective performance; OP: objective performance; rCDHEA3: the cortisol to DHEA ratio at post-task 15 minutes.

Table 2 Correlations between objective performance and significant factors $(N=43)$.

\begin{tabular}{llllllll}
\hline & OP & STAIt & Appraisal & COPE & STAIs & NA & rCDHEA3 \\
\hline OP & 1 & & & & & \\
STAIt & $-0.37^{*}$ & 1 & & & & \\
Appraisal & $-0.36^{*}$ & $0.47^{* *}$ & 1 & & & & \\
COPE & $-0.57^{* *}$ & $0.56^{* *}$ & $0.50^{* *}$ & 1 & & & \\
STAIs & $-0.50^{* *}$ & $0.56^{* *}$ & $0.68^{* *}$ & $0.71^{* *}$ & 1 & & \\
NA & $-0.46^{* *}$ & $0.59^{* *}$ & $0.65^{* *}$ & $0.71^{* *}$ & $0.86^{* *}$ & 1 & $0.38^{*}$ \\
rCDHEA3 & $-0.30^{*}$ & 0.20 & 0.22 & $0.33^{*}$ & 0.25 & 1 \\
\hline
\end{tabular}

OP: objective performance; STAIt: trait anxiety; Appraisal: ratio of threat appraisal/challenge appraisal; COPE: dysfunctional coping; STAIs: post-task states anxiety; NA: post-task negative affect; rCDHEA3: ratio of cortisol/DHEA at post-task 15 minutes.

${ }^{*} P<0.05 .{ }^{* *} P<0.01$.

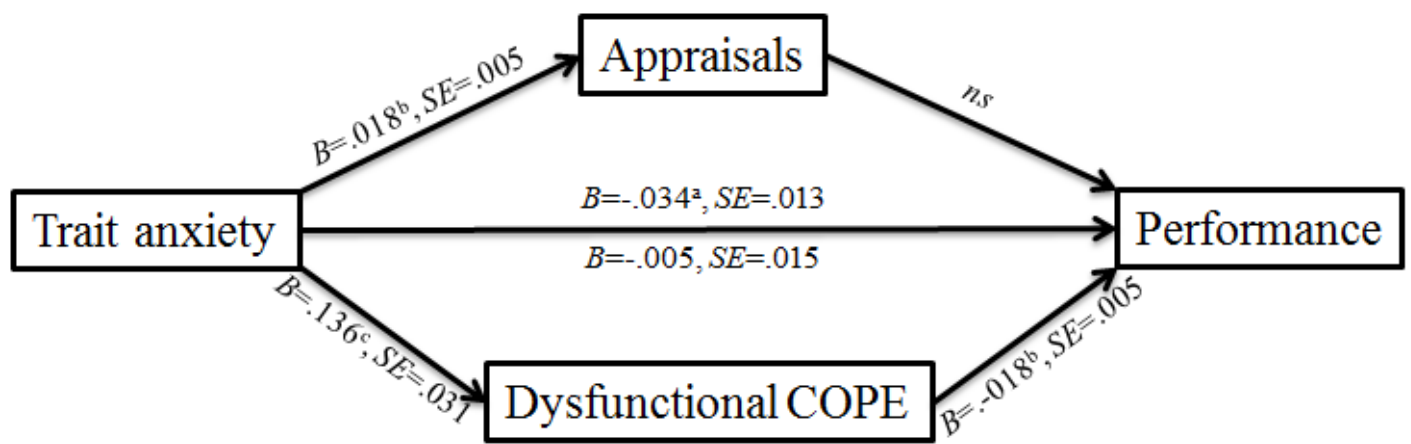

Fig. 2 Mediation effect between trait anxiety, appraisals, dysfunctional coping and objective performance. ${ }^{\mathrm{a}} P<0.05,{ }^{\mathrm{b}} P<0.01,{ }^{\mathrm{c}} P<0.001$.

dysfunctional coping significantly predicted $=0.72$ respectively (Fig. 2). The difference between performance score whilst appraisals did not the total and direct effects of trait anxiety and significantly predict performance.

The direct and total effects of trait anxiety on performance score were $-0.03, P<0.05$ and $-0.005, P$ performance score was accounted for by the total indirect effect of coping and appraisals. Examination of the specific indirect mediating effects indicated that 
only dysfunctional coping contributed to the total indirect mediating effect with accounting for .02 (95\% $\mathrm{CI}=[-0.044,-0.009])$ of the total effect.

\section{Discussion}

The present study aimed to investigate acute stress responses by multiple assessments. To date, acute stress responses have been assessed mostly through only physiological and psychological measures and few publications reported collective results with multidimensional methods [6, 15]. Only Schneider [15] has examined multiple stress responses (i.e. psychological, physiological and the performance) using a mental arithmetic task. However, results observed with this type of stressor may not generalize to public speaking performances.

Our results showed that among the multivariable factors, psychological factors seemed to be the most sensitive to stress performance. These factors included trait anxiety, negative emotional experience, ratio of threat/challenge appraisals and dysfunctional coping (i.e. behaviour disengagement and self-blame). Those factors tend to link with vulnerable stress response and performance. According to the literature, anxiety and negative emotional experiences are associated with physiological stress response [61, 62], but also contribute to worsen performance [19, 20]. In line with our hypothesis, trait anxiety tends to be a risk factor for performance. With regards to the cognitive component, our results are consistent with previous studies since threat appraisal was associated with more negative emotions and poor performance [28]. Literature also supports the view that dysfunctional coping contributes to bad academic performances [30].

Our study highlighted a negative association between the cortisol to DHEA ratio (i.e. 15 minutes after the task) and objective performance. Our results indicated that this endocrine imbalance was associated with a bad performance, which was partially supported by the previous founding $[3,5]$. To date, evidence has been sparse in showing a direct link between the cortisol level, or cortisol to DHEA ratio and stress performance. Literature supports that an elevated cortisol level is associated with a poor TSST related decision-making performance [5]. In another study, higher stress and high cortisol levels were associated with low acadamic performances, i.e. examination scores [3]. However, little evidence can be found with regards to a public speaking performance. In the current study, this imbalance was also associated with two components: dysfunctional coping strategies (i.e. behavioural disengagement and self-blame) and negative emotional states. As Nicholls et al. [63] pointed out, emotions and coping are constructs that are associated with performance satisfaction. Studies also suggested that these two components play a critical role in academic achievement and stress performance [2, 30]. Similarly, Izawa et al. [34] considered the links between cortisol/DHEA ratio during the TSST and negative mood. They reported negative mood states in association with lower DHEA and elevated cortisol/DHEA ratio. Our results suggest that those negative emotions and the use of dysfunctional coping strategies in the stressful situation can be associated with the physiological response and thus may contribute to the failure of performance. In the present study, we did not find any links between the speaking performance and cortisol response, nor DHEA. Nevertheless, our results suggest the tendency that the endocrine balance may be more favourable as a biomarker in explaining stress performance.

Behavioural responses have been assessed in response to a psychosocial stressor. In contrast with our hypothesis, we did not observe the Contraction index associated with speaking performance. The evaluation of objective speaking performance may be not sensitive enough to put forward this link at the global level of the whole session. Instead, it might be relevant to consider fine-grain analyses of episodes during which participants are perceived as being 
stressed by experts.

Furthermore, we observed that the negative association between trait anxiety and performance score was totally mediated by threat/challenge appraisals and dysfunctional coping. Examination of the specific indirect mediating effects showed that only dysfunctional coping contributed to the total indirect mediating effect. This result suggests the possible mechanism underlying the relationship between trait anxiety and performance. It indicates that trait anxiety may lead to poor performance though the cognitive function, but more importantly, through the inability to manage stress, i.e. dysfunctional coping. As we pointed above, when people are not able to cope well with the stressful situation, they tend to have more negative emotional experiences, which are also reflected in the physiological response such as the endocrine imbalance (i.e. the cortisol to DHEA ratio), thus contributing to the failure of the public speaking performance. Villada et al. [64] referred to the importance of traits anxiety and coping styles (i.e. emotions and mental disengagement) in understanding the overall integrative psychobiological responsiveness to social stress. Similar mediation was highlighted between neuroticism and psychological distress by avoidance, religious coping and behavioural disengagement [44], pointing to the negative impact of such dysfunctional coping strategies for psychological distress among individuals who are sensitive to stress.

In terms of limitation, the definition of "student" is a bit broad. With the aim to help more people find a way to perform better in public speaking, we have recruited students all over the university campus. In the future study, we may also be able to pay more attention to students at certain age, sex and specialty, and better with a larger sample. There is also a common limitation concerning laboratory-induced stressors. More pronounced stress and changes related to performance were observed in real-world stressful situation than in laboratory conditions [65]. Indeed, there might be a disconnection between laboratory and real-world operational studies and outcomes, emphasizing the real-world research designs should never be replaced by laboratory experimentation paradigms [66]. Moreover, concerning the assessor-rated performance, different studies uses different criteria so that standardized criteria in evaluating public speaking performance is lacking. We used the global score given by three evaluators on the quality of the content, the way to express, and the answers in the feedback session. There may be more results linking with these different scores, which should be examined in future studies.

To sum up, our results add evidence to the literature with regard to a multidimensional way to study human stress response. With such knowledge of which factors may contribute to a failure of public speaking performance, practitioners may gain a broad vision and better help individuals, especially those anxious ones prepare for a real-life stressful situation such as job interviews. Certain personality traits may also help predict if people are at risk of developing more stressful feelings and be maladapted to such situation so interventions can be made accordingly. More importantly, practitioners may help people learn to cope effectively to the stressful situation in a positive way, e.g., using functional coping strategies. And finally, training to improve their confidence and lower their anxiety would also be helpful. Anderson et al. [67] conducted a cognitive-behavioural treatment to provide anxiety management training, and it may reduce public-speaking anxiety.

\section{Acknowledgement}

This work was supported by the French national multidisciplinary project ANR COMPARSE under Grant <ANR-11-EMCO-0007>.

\section{References}

[1] Laborde, S., Lautenbach, F., Allen, M. S., Herbert, C., and Achtzehn, S. 2014. "The Role of Trait Emotional Intelligence in Emotion Regulation and Performance 
under Pressure." Personality and Individual Differences 57 (4): 43-7.

[2] Ng, V., Koh, D., and Chia, S. E. 2003. "Examination Stress, Salivary Cortisol, and Academic Performance." Psychological Reports 93 (3 Pt 2): 1133-4.

[3] Pruessner, M., Pruessner, J. C., Hellhammer, D. H., Bruce Pike, G., and Lupien, S. J. 2007. "The Associations among Hippocampal Volume, Cortisol Reactivity, and Memory Performance in Healthy Young Men.” Psychiatry Research 155 (1): 1-10.

[4] Van den Bos, R., Harteveld, M., and Stoop, H. 2009. "Stress and Decision-making in Humans: Performance Is Related to Cortisol Reactivity, Albeit Differently in Men and Women." Psychoneuroendocrinology 34 (10): 1449-58.

[5] Campbell, J., and Ehlert, U. 2012. "Acute Psychosocial Stress: Does the Emotional Stress Response Correspond with Physiological Responses?” Psychoneuroendocrinology 37 (8): 1111-34.

[6] Dickerson, S. S., and Kemeny, M. E. 2004. "Acute Stressors and Cortisol Responses: A Theoretical Integration and Synthesis of Laboratory Research." Psychological Bulletin 130 (3): 355-91.

[7] Jönsson, P., Wallergård, M., Osterberg, K., Hansen, A. M., Johansson, G., and Karlson, B. 2010. "Cardiovascular and Cortisol Reactivity and Habituation to a Virtual Reality Version of the Trier Social Stress Test: A Pilot Study.” Psychoneuroendocrinology 35 (9): 1397-403.

[8] Birkett, M. A. 2011. "The Trier Social Stress Test Protocol for Inducing Psychological Stress." Journal of Visualized Experiments 64 (56): 998-1011.

[9] Schommer, N. C., Hellhammer, D. H., and Kirschbaum, C. 2002. "Dissociation between Reactivity of the Hypothalamus-pituitary-adrenal Axis and the Sympathetic-adrenal-medullary System to Repeated Psychosocial Stress." Psychosomatic Medicine 65 (3): 450-60.

[10] Al'Absi, M., Bongard, S., Buchanan, T., Pincomb, G. A., Licinio, J., and Lovallo, W. R. 1997. "Cardiovascular and Neuroendocrine Adjustment to Public Speaking and Mental Arithmetic Stressors.” Psychophysiology 34 (3): 266-75.

[11] Hua, J., Le Scanff, C., Larue, J., José, F., Martin, J. C., Devillers, L., and Filaire, E. 2014. "Global Stress Response during a Social Stress Test: Impact of Alexithymia and Its Subfactors.” Psychoneuroendocrinology 50 (10): 53-61.

[12] Allen, A. P., Kennedy, P. J., Cryan, J. F., Dinan, T. G., and Clarke, G. 2014. "Biological and Psychological Markers of Stress in Humans: Focus on the Trier Social Stress Test." Neuroscience \& Biobehavioral Reviews 38: 94-124.
[13] Suls, J. 2001. “Affect, Sress, and Personality.” Handbook of Affect and Social Cognition. Mahwah: Lawrence Erlbaum Associates Publishers, Xviii, 392-409.

[14] Schneider, T. R. 2004. "The Role of Neuroticism on Psychological and Physiological Stress Responses." Journal of Experimental Social Psychology 40 (6): 795-804.

[15] Phillips, A. C., Carroll, D., Burns, V. E., and Drayson, M. 2005. "Neuroticism, Cortisol Reactivity, and Antibody Response to Vaccination." Psychophysiology 42 (2): 232-8.

[16] Bibbey, A., Carroll, D., Roseboom, T. J., Phillips, A. C., and de Rooij, S. R. 2013. "Personality and Physiological Reactions to Acute Psychological Stress.” International Journal of Psychophysiology: Official Journal of the International Organization of Psychophysiology 90 (1): 28-36.

[17] Oswald, L. M., Zandi, P., Nestadt, G., Potash, J. B., Kalaydjian, A. E., and Wand, G. S. 2006. "Relationship between Cortisol Responses to Stress and Personality." Neuropsychopharmacology: Official Publication of the American College of Neuropsychopharmacology 31 (7): 1583-91.

[18] Owens, M., Stevenson, J., Norgate, R., and Hadwin, J. A. 2008. "Processing Efficiency Theory in Children: Working Memory As a Mediator between Trait Anxiety and Academic Performance." Anxiety, Stress, and Coping 21 (4): 417-30.

[19] Kenny, D. T., and Osborne, M. S. 2006. "Music Performance Anxiety: New Insights from Young Musicians." Advances in Cognitive Psychology 2 (2): 103-12.

[20] Patel, D. R., Omar, H., and Terry, M. 2010. "Sport-Related Performance Anxiety in Young Female Athletes." Journal of Pediatric and Adolescent Gynecology 23 (6): 325-35.

[21] Raglin, J. S. 1992. "Anxiety and Sport Performance." Exercise and Sport Sciences Reviews 20 (4): 243-74.

[22] Vitasari, P., Wahab, M. N. A., Herawan, T., Sinnadurai, S. K., Othman, A., and Awang, M. G. 2011. "Assessing of Physiological Arousal and Cognitive Anxiety toward Academic Performance: The Application of Catastrophe Model." Procedia-Social and Behavioral Sciences 30 (10): 615-9.

[23] Gonzalez-Bono, E., Moya-Albiol, L., Salvador, A., Carrillo, E., Ricarte, J., and Gomez-Amor, J. 2002. "Anticipatory Autonomic Response to a Public Speaking Task in Women.” Biological Psychology 60 (1): 37-49.

[24] Saab, P. G., Matthews, K. A., Stoney, C. M., and McDonald, R. H. 1989. "Premenopausal and Postmenopausal Women Differ in Their Cardiovascular and Neuroendocrine Responses to Behavioral Stressors." 
Psychophysiology 26 (3): 270-80.

[25] Folkman, S., and Lazarus, R. S. 1985. "If It Changes It Must be a Process: Study of Emotion and Coping during Three Stages of a College Examination." Journal of Personality and Social Psychology 48 (1): 150-70.

[26] Tomaka, J., Blascovich, J., Kelsey, R. M., and Leitten, C. L. 1993. "Subjective, Physiological, and Behavioral Effects of Threat and Challenge Appraisal." Journal of Personality and Social Psychology 65 (2): 248-60.

[27] Baggett, H. L., Saab, P. G., and Carver, C. S. 1996. "Appraisal, Coping, Task Performance, and Cardiovascular Responses During the Evaluated Speaking Task." Personality and Social Psychology Bulletin 22 (5): 483-94.

[28] Brown, G. K., and Nicassio, P. M. 1987. "Development of a Questionnaire for the Assessment of Active and Passive Coping Strategies in Chronic Pain Patients." Pain 31 (1): 53-64.

[29] Gallagher, D. J. 1996. "Personality, Coping, and Objective Outcomes: Extraversion, Neuroticism, Coping Styles, and Academic Performance." Personality and Individual Differences 21 (3): 421-9.

[30] Shirotsuki, K., Izawa, S., Sugaya, N., Yamada, K. C., Ogawa, N., Ouchi, Y., Nagano, Y., and Nomura, S. 2009. "Salivary Cortisol and DHEA Reactivity to Psychosocial Stress in Socially Anxious Males." International Journal of Psychophysiology: Official Journal of the International Organization of Psychophysiology 72 (2): 198-203.

[31] Lennartsson, A. K., Kushnir, M. M., Bergquist, J., and Jonsdottir, I. H. 2012. "DHEA and DHEA-S Response to Acute Psychosocial Stress in Healthy Men and Women." Biological Psychology 90 (2): 143-9.

[32] Do Vale, S., Martin Martins, J., Fagundes, M. J., and do Carmo, I. $2011 . \quad$ "Plasma Dehydroepiandrosterone-sulphate Is Related to Personality and Stress Response." Neuro Endocrinology Letters 32 (4): 442-8.

[33] Izawa, S., Sugaya, N., Shirotsuki, K., Yamada, K. C., Ogawa, N., Ouchi, Y., Nagano, Y., Suzuki, K., and Nomura, S. 2008. "Salivary Dehydroepiandrosterone Secretion in Response to Acute Psychosocial Stress and Its Correlations with Biological and Psychological Changes." Biological Psychology 79 (3): 294-8.

[34] Van Niekerk, J. K., Huppert, F. A., and Herbert, J. 2001. "Salivary Cortisol and DHEA: Association with Measures of Cognition and Well-being in Normal Older Men, and Effects of Three Months of DHEA Supplementation." Psychoneuroendocrinology 26 (6): 591-612.

[35] Blauer, K. L., Poth, M., Rogers, W. M., and Bernton, E. W. 1991. "Dehydroepiandrosterone Antagonizes the
Suppressive Effects of Dexamethasone on Lymphocyte Proliferation." Endocrinology 129 (6): 3174-9.

[36] Morgan, C. A., Southwick, S., Hazlett, G., Rasmusson, A., Hoyt, G., Zimolo, Z., and Charney, D. 2004. "Relationships among Plasma Dehydroepiandrosterone Sulfate and Cortisol Levels, Symptoms of Dissociation, and Objective Performance in Humans Exposed to Acute Stress." Archives of General Psychiatry 61 (8): 819-25.

[37] Bonardi, A. I., and Truck, H. A. 2006. "Building Fuzzy Rules in an Emotion Detector." 11th International Conference on Information Processing and Management of Uncertainty in Knowledge-Based Systems (IPMU'2006).

[38] Devillers, L., Vidrascu, L., and Lamel, L. 2005. "Challenges in Real-life Emotion Annotation and Machine Learning based Detection." Neural Networks 18 (4): 407-22.

[39] Burgoon, J., Jensen, M., Meservy, T. O., Kruse, J., and Nunamaker, J. 2005. "Augmenting Human Identification of Emotional States in Video University of Arizona." Computer and Information Science 6 (2): 123-50.

[40] Hagenaars, M. A., and van Minnen, A. 2005. "The Effect of Fear on Paralinguistic Aspects of Speech in Patients with Panic Disorder with Agoraphobia." Journal of Anxiety Disorders 19 (5): 521-37.

[41] Ruiz, R., Absil, E., Harmegnies, B., Legros, C., and Poch, D. 1996. "Time- and Spectrum-related Variabilities in Stressed Speech under Laboratory and Real Conditions." Speech Communication 20: 111-29.

[42] Camurri, A., Lagerlöf, I., and Volpe, G. 2003. "Recognizing Emotion from Dance Movement: Comparison of Spectator Recognition and Automated Techniques." International Journal of Human-Computer Studies 59 (1-2): 213-25.

[43] Panayiotou, G., Kokkinos, C. M., and Kapsou, M. 2002. "Indirect and Direct Associations between Personality and Psychological Distress Mediated by Dispositional Coping." The Journal of Psychology 148 (5): 549-67.

[44] John, R. L., Donahue, O. P., and Kentle, E. M. 1991. The Big Five Inventory-Versions $4 a$ and 54. Berkeley, CA: University of California, Berkeley, Institute of Personality and Social Research.

[45] Plaisant, O., Courtois, R., Réveillère, C., Mendelsohn, G. A., and John, O. P. 2010. "Factorial Analysis Validation of the Big Five Inventory Français (BFI-Fr). Convergent Analysis with NEO-PI-R." Medicine, Psychological, Psychiatric Review 168 (2): 97-106. (in French)

[46] Spielberger, C. D., Jacobs, G., Russell, S., and Crane, R. S. 1983. "Assessment of Anger: The State-Trait Anger Scale." Advances in Personality Assessment 2: 159-87.

[47] Bruchon-Schweitzer, M., and Paulhan, I. 1993. The State-Trait Anxiety Inventory, Form Y. Paris: Editions of 
Applied Psychological Center. (in French)

[48] Watson, D., Clark, L. A., and Tellegen, A. 1988. "Development and Validation of Brief Measures of Positive and Negative Affect: the PANAS Scales." Journal of Personality and Social Psychology 54 (6): 1063-70.

[49] Gaudreau, P. 2007. "Toward a French Version of the PANAS: Principal Components Analyses before, during, and after a Sport Competition." Presented at the International Conference of the French Society of Sport Psychology, Paris. (in French)

[50] Vermeulen, N., Corneille, O., and Luminet, O. 2007. “A Mood Moderation of the Extrinsic Affective Simon Task." European Journal of Personality 21: 359-69.

[51] Carver, C. S. 1997. "You Want to Measure Coping but Your Protocol's Too Long: Consider the Brief COPE." International Journal of Behavioral Medicine 4 (1): 92-100.

[52] Muller, L., and Spitz, E. 2003. "Muldimensionnal Evaluation of Coping: Validation of the Brief Cope on a French Population." Brain: Psychiatric Review, Clinical, Biological and Therapeutic 29 (6): 507-18. (in French)

[53] Schneider, T. R., Rench, T. A., Lyons, J. B., and Riffle, R. R. 2012. "The Influence of Neuroticism, Extraversion and Openness on Stress Responses." Stress and Health: Journal of the International Society for the Investigation of Stress 28 (2): 102-10.

[54] Fydrich, T., Chambless, D. L., Perry, K. J., Buergener, F., and Beazley, M. B. 1998. "Behavioral Assessment of Social Performance: A Rating System for Social Phobia." Behaviour Research and Therapy 36 (10): 995-1010.

[55] Harb, G. C., Eng, W., Zaider, T., and Heimberg, R. G. 2003. "Behavioral Assessment of Public-Speaking Anxiety Using a Modified Version of the Social Performance Rating Scale." Behaviour Research and Therapy 41 (11): 1373-80.

[56] Alonso, M., Varkevisser, M., Hekkert, P., and Keyson, D. 2007. Exploring Manipulative Hand Movements During a Stressful Condition. Berlin: Springer Berlin Heidelberg, 998-1011.

[57] Papacosta, E., and Nassis, G. P. 2011. "Saliva as a Tool for Monitoring Steroid, Peptide and Immune Markers in
Sport and Exercise Science." Journal of Science and Medicine in Sport 14 (5): 424-34.

[58] Preacher, K. J., and Hayes, A. F. 2004. "SPSS and SAS Procedures for Estimating Indirect Effects in Simple Mediation Models." Behavior Research Methods, Instruments, and Computers 36: 717-31.

[59] Preacher, K. J., and Hayes, A. F. 2008. “Asymptotic and Resampling Strategies for Assessing and Comparing Indirect Effects in Multiple Mediator Models." Behavior Research Methods 40: 879-91.

[60] Carrillo, E., Moya-Albiol, L., González-Bono, E., Salvador, A., Ricarte, J., and Gómez-Amor, J. 2001. "Gender Differences in Cardiovascular and Electrodermal Responses to Public Speaking Task: The Role of Anxiety and Mood States." International Journal of Psychophysiology: Official Journal of the International Organization of Psychophysiology 42 (3): 253-64.

[61] Chida, Y., and Hamer, M. 2008. "Chronic Psychosocial Factors and Acute Physiological Responses to Laboratory-induced Stress in Healthy Populations: A Quantitative Review of 30 Years of Investigations." Psychological Bulletin 134 (6): 829-85.

[62] Nicholls, A. R., Polman, R. C. J., and Levy, A. R. 2012. "A Path Analysis of Stress Appraisals, Emotions, Coping, and Performance Satisfaction among Athletes." Psychology of Sport and Exercise 13 (3): 263-70.

[63] Villada, C., Hidalgo, V., Almela, M., and Salvador, A. 2014. "Individual Differences in the Psychobiological Response to Psychosocial Stress (Trier Social Stress Test): The Relevance of Trait Anxiety and Coping Styles." Stress and Health: Journal of the International Society for the Investigation of Stress 64 (4): 998-1011.

[64] Wilson, G. P., Skelly, J., and Purvis, B. 1999. "Reactions to Emergency Situations in Actual and Simulated Flight." Human Performance in Extreme Environments 4 (2): 34-45.

[65] Staal, M. A. 2004. "Stress, Cognition, and Human Performance: A Literature Review and Conceptual Framework." NASA Technical Memorandum, August.

[66] Anderson, P. L., Zimand, E., Hodges, L. F., and Rothbaum, B. O. 2005. "Cognitive Behavioral Therapy for Public-Speaking Anxiety Using Virtual Reality for Exposure." Depression and Anxiety 22 (3): 156-8. 